\title{
Calculation of the Mass of the Universe, the Radius of the Universe, the Age of the Universe and the Quantum of Speed
}

\author{
Claude Mercier \\ Independent Researcher, Baie-Comeau, Canada \\ Email: claude.mercier@gctda.com
}

How to cite this paper: Mercier, C. (2019) Calculation of the Mass of the Universe, the Radius of the Universe, the Age of the Universe and the Quantum of Speed. Journal of Modern Physics, 10, 980-1001. https://doi.org/10.4236/jmp.2019.108065

Received: June 15, 2019

Accepted: July 16, 2019

Published: July 19, 2019

Copyright $\odot 2019$ by author(s) and Scientific Research Publishing Inc. This work is licensed under the Creative Commons Attribution International License (CC BY 4.0).

http://creativecommons.org/licenses/by/4.0/

\begin{abstract}
The universe is vast and when we look at the sky, its parameters (dimensions, mass, and age) seems limitless. Lemaitre proposed that the universe began from a primeval-atom [1] which was later ironically nicknamed by Hoyle "Big Bang" in a BBC broadcast in 1949 [2]. From general relativity, Einstein proposed a cosmological model [3] with a spatially finite universe. He assumed a uniform distribution of matter in a huge 4-D sphere. Even if his equations were showing that the universe was either contracting or expanding, Einstein introduced the "cosmological constant" in his equation to force the universe to be static (being consistent with the general way of thinking of his time). In 1929, from observations of galaxies, Hubble found that the universe was expanding. From that moment, Einstein discarded his cosmological constant as an unnecessary fudge factor. Many cosmological models have been built over time. Each of them excels in explaining some aspects of the universe. We consider that the global topology of the universe is not known, but making the assumptions that it is relatively homogenous and isotropic, its extrapolated local topology leads us to some global "apparent" parameters. From our new cosmological model, we calculate the main parameters of the universe which are its apparent mass $m_{u}$ its apparent curving radius $R_{u}$, its apparent age $T_{u}$ and the "quantum of speed" $\mathcal{E}_{r}$. The quantum of speed is a new notion in physics. It is the smallest speed increment that may exist. For metrology purposes, we calculate these parameters from the most precise physics' parameters available.
\end{abstract}

$$
\begin{gathered}
m_{u} \approx 1.73 \times 10^{53} \mathrm{~kg}, \quad R_{u} \approx 1.28 \times 10^{26} \mathrm{~m}, \\
T_{u} \approx 13.65 \times 10^{9} \text { years, }, \varepsilon_{v} \approx 2.38 \times 10^{-114} \mathrm{~m} \cdot \mathrm{s}^{-1}
\end{gathered}
$$

\section{Keywords}

Mass of the Universe, Hubble Radius, Age of the Universe, Quantum of 
Speed, Dirac Large Numbers Hypothesis

\section{Introduction}

Our universe has astronomic dimensions (mass, radius, and age) that borders limitless for humans. It is also expanding [4]. Astrophysicists always try to describe our universe more accurately according to observations. Our telescopes are more and more powerful which allow us to see further every day. In a previous article [5] we made a new cosmological model from which we can deduce different parameters and dimensions. Because these different dimensions of the universe are directly linked through Dirac's large numbers to infinitely small [6] [7], it is possible to make different calculations to allow finding all the exact values of these dimensions.

This article shows different ways to calculate the apparent mass $m_{u}$ of the universe, the apparent radius $R_{u}$ of curvature of the universe and the age of the universe. We want also to make conscious that these values are obtained from an observer at rest, at the center of mass of the universe. Nevertheless, if the observer is traveling on a photon, his point of view will be totally different. For this reason, we will show the notion of quantum of speed. With this notion, we will see that there is an infinitely small difference between the real speed of light and the speed limit that we call the speed of light in vacuum $c$. For most applications, the real speed of light and the speed limit are approximately the same.

We will then show different links between the infinitely large numbers of the universe and the infinitely small numbers of the universe thanks to Dirac's hypothesis [6] [7].

\section{Values of Physics Parameters}

We will use the concise form of notation to display tolerances (2.736 (17) K will mean $2.736 \pm 0.017 \mathrm{~K})$. The following physics parameters are from CODATA 2014 [8].

- Speed of light in vacuum $c \approx 299792458 \mathrm{~m} \cdot \mathrm{s}^{-1}$

- Electric constant in vacuum $\varepsilon_{0} \approx 8.854187817 \times 10^{-12} \mathrm{~F} \cdot \mathrm{m}^{-1}$

- Magnetic constant in vacuum $\mu_{0} \approx 4 \pi \times 10^{8} \mathrm{~N} \cdot \mathrm{A}^{-2}$

- Planck constant $h \approx 6.626070040(81) \times 10^{-34} \mathrm{~J} \cdot \mathrm{s}$

- Planck length $L_{p} \approx 1.616229(38) \times 10^{-35} \mathrm{~m}$

- Planck time $t_{p} \approx 5.39116(13) \times 10^{-44} \mathrm{~s}$

- Planck mass $m_{p} \approx 2.176470(51) \times 10^{-8} \mathrm{~kg}$

- Universal gravitational constant $G \approx 6.67408(31) \times 10^{-11} \mathrm{~m}^{3} \cdot \mathrm{kg}^{-1} \cdot \mathrm{s}^{-2}$

- Electron charge $q_{e} \approx-1.6021766208(98) \times 10^{-19} \mathrm{C}$

- Electron mass $m_{e} \approx 9.10938356(11) \times 10^{-31} \mathrm{~kg}$

- Classical electron radius $r_{e} \approx 2.8179403227(19) \times 10^{-15} \mathrm{~m}$

- Rydberg constant $R_{\infty} \approx 10973731.568508(65) \mathrm{m}^{-1}$ 


\section{Apparent Mass of the Universe}

Let us enumerate different ways to calculate the apparent mass of the universe $m_{u}$. This mass includes every type of mass (baryonic and dark mass) and the mass associated with all types of energy (photons, dark energy, etc.).

We prefer to talk about the "apparent mass of the universe" instead of talking about the "mass of the universe" because its apparent value is seen from our point of view in the universe. For an observer located somewhere else, the observed value may be different. Please refer to our section talking about the quantum of speed to deepen your notion of "appearance" for the different parameters of the universe.

\subsection{Calculation of $m_{u}$ Using the Principle of Conservation of Momentum}

Let us use the principle of conservation of momentum applied to the universe which says that a force $F$ applied during a time $\Delta t$ will move a mass $m_{u}$ (the apparent mass of the universe) by increasing its speed by $\Delta v$. The luminous universe is expanding at the speed of light in vacuum $c$. Therefore, we can consider that in Equation (1), $\Delta v=c$.

$$
F \Delta t=m_{u} \Delta v \rightarrow F=\frac{m_{u} \Delta v}{\Delta t} \rightarrow F=\frac{m_{u} c}{\Delta t}
$$

Let us suppose that the universe is expanding for a time $\Delta t$ equal to the apparent age of the universe $T_{u}$. This value is given by the inverse of the Hubble constant which is about $H_{0} \approx 72.1 \mathrm{~km} \cdot \mathrm{s}^{-1} \cdot \mathrm{MParsec}^{-1}$ [5] [9]. Let us note that 1 MParsec $=3.085677581 \times 10^{22} \mathrm{~m}$.

$$
\Delta t=T_{u}=\frac{1}{H_{0}} \approx 13.65 \times 10^{9} \text { years }
$$

If we look at the universe as a whole, and if we use Newton's universal attraction equation to calculate the force $F$ that the universe of apparent radius $R_{u}$ applies on its own mass $m_{u}$, we get Equation (3). The constant $G$ is the universal gravitational constant.

$$
F=\frac{G m_{u}^{2}}{R_{u}^{2}}
$$

In Equation (3), the value of $R_{u}$ is the apparent radius of curvature of the universe.

$$
R_{u}=c T_{u}=\frac{c}{H_{0}} \approx 1.28 \times 10^{26} \mathrm{~m}
$$

Let us equate the forces $F$ from Equation (1) and (3). Once we simplify and use Equation (2) and Equation (4), we get Equation (5).

$$
m_{u}=\frac{c^{3}}{G H_{0}} \approx 1.73 \times 10^{53} \mathrm{~kg}
$$

We get an equation which is the same as Carvalho [10]. 


\subsection{Calculation of $m_{u}$ Using the Principle of Conservation of Energy}

Let us use the principle of conservation of Energy to find the apparent mass $m_{u}$ of the universe. Initially, at the Big Bang, there was no movement yet. All the energy contained in the universe was contained in the mass $m_{u}$ of the universe. The total amount of energy $E$ contained in the universe is given by Einstein formula.

$$
E=m_{u} c^{2}
$$

Since the universe is expanding, the initial energy is converted to potential energy through a gravitational force $F$ applied on a distance $R_{u}$ (see Equation (4)) from the center of mass of the universe. Let us use Newton's law.

$$
F=\frac{G m^{2}}{R_{u}^{2}} \rightarrow E=F R_{u}=\frac{G m^{2}}{R_{u}}
$$

By equating Equation (6) and Equation (7), and using Equation (4), we get Equation (8).

$$
m_{u} c^{2}=\frac{G m_{u}^{2}}{R_{u}} \text { where } R_{u}=\frac{c}{H_{0}}
$$

Isolating the apparent mass of the universe $m_{u}$ from Equation (8), we get Equation (9).

$$
m_{u}=\frac{c^{2} R_{u}}{G}=\frac{c^{3}}{G H_{0}} \approx 1.73 \times 10^{53} \mathrm{~kg}
$$

One will notice that this is the same equation as Equation (5).

\subsection{Calculation of $m_{u}$ Using the Planck Mass $m_{p}$}

Let us calculate the apparent mass of the universe $m_{u}$ by using the Planck mass $m_{p}$. By definition, the Plank mass is defined by Equation (10) where $h$ is the Planck constant, $c$ is the speed of light, and $G$ is the universal gravitational constant.

$$
m_{p}=\sqrt{\frac{h c^{3}}{2 \pi G}} \approx 2.18 \times 10^{-8} \mathrm{~kg}
$$

Let us define $m_{p h}$ as being the mass associated with the lowest energy photon in the universe [5]. When we look at the energy of a wavelength $\lambda$, the energy is at its lowest level when $\lambda$ is the largest. The largest dimension of the universe is its apparent circumference. Therefore, we can associate a mass $m_{p h}$ to a photon of wavelength $\lambda=2 \pi R_{u}$ where $R_{u}$ is the apparent radius of the universe.

$$
m_{p h}=\frac{h c}{\lambda c^{2}}=\frac{h c}{2 \pi R_{u} c^{2}}=\frac{h H_{0}}{2 \pi c^{2}} \approx 2.74 \times 10^{-69} \mathrm{~kg}
$$

The Plank mass $m_{p}$ is the geometric average between the smallest mass $m_{p h}$ associated with the lowest energy photon and the biggest mass which is without any doubt the apparent mass of the universe $m_{u}$. From this fact, we get the following Equation. 


$$
m_{p}=\sqrt{m_{p h} m_{u}} \approx 2.17 \times 10^{-8} \mathrm{~kg}
$$

Equating Equations (12) and (10), and using Equation (11), we get $m_{u}$.

$$
m_{u}=\frac{c^{3}}{G H_{0}} \approx 1.73 \times 10^{53} \mathrm{~kg}
$$

Again, we get, without any surprise, the same equation as Equation (5).

\subsection{Calculation of $m_{u}$ Using the Energy of a Photon}

We will calculate the apparent mass $m_{u}$ of the universe by equating the gravitational energy of a photon with the mass-energy contained in a photon (being a corpuscle) [5]. Let us associate a mass $m_{p h}$ (like in Equation (11)) with a photon of the lowest energy [5] that is at the periphery of the luminous universe (with a wavelength $\lambda$ equal to the apparent circumference of the universe $\lambda=2 \pi R_{u}$ ). Then, if we place this photon at the periphery of the luminous universe, it will have an $E_{g}$ gravitational energy.

$$
E_{g}=\frac{G m_{u} m_{p h}}{R_{u}}
$$

According to the special relativity, the mass-energy associated with this photon is $E_{m}$.

$$
E_{m}=m_{p h} c^{2}
$$

By equating Equations (14) and (15), replacing $R_{u}$ with Equation (4), and isolating $m_{u}$, we get the same equation as Equation (5).

$$
m_{u}=\frac{c^{3}}{G H_{0}} \approx 1.73 \times 10^{53} \mathrm{~kg}
$$

\subsection{Calculation of $m_{u}$ as a Function of the Classical Electron Radius $r_{e}$}

Recently, with a new cosmological model, the precise values of the universal gravitational constant $G$ and of the Hubble constant $H_{0}$ have been found [5] as a function of the classical electron radius $r_{e}$, the mass of the electron $m_{e}$, the fine-structure constant $\alpha$, and $\beta$ (see Equation (19)). Let us use Equations (17) and (18) to evaluate precisely $m_{u}$ (for metrology purposes).

$$
\begin{gathered}
G=\frac{c^{2} r_{e} \alpha^{20}}{m_{e} \beta} \approx 6.673229809(86) \times 10^{-11} \mathrm{~m}^{3} \cdot \mathrm{kg}^{-1} \cdot \mathrm{s}^{-2} \\
H_{0}=\frac{c \alpha^{19} \sqrt{\beta}}{r_{e}} \approx 72.09548580(32) \mathrm{km} \cdot \mathrm{s}^{-1} \cdot \mathrm{MParsec}^{-1}
\end{gathered}
$$

In these two equations, $\beta$ is defined as the ratio between the expansion speed of the material universe and the expansion speed of the luminous universe. According to our model, the material universe is embedded in a luminous universe, both being spherical and expanding with a speed proportional to their radius [5].

$$
\beta=3-\sqrt{5} \approx 0.76
$$


with his special relativity theory, Einstein showed that if we accelerate any mass at rest $m_{0}$ to a speed $v$, its energy $E$ would increase because of the Lorentz factor (the square root in Equation (20)) [11]. If $v$ tends towards $\mathcal{c}$, the energy $E$ would tend towards infinity, which is impossible since it cannot get more energy than there is available in the universe. Therefore, $v$ must be slower than $c$. It also implies that the expansion speed of the material universe is slower (explaining $\beta$ in Equation (19)) than its luminous counterpart which is expanding at the speed of light (which is $c$ for now). In Equation (20), we show what would happen if we expand Einstein's formula in a series. The first term of the series is the energy at rest and the second one is the kinetic energy which is used in Newton's classical mechanics [12].

$$
E=m c^{2}=\frac{m_{0} c^{2}}{\sqrt{1-\frac{v^{2}}{c^{2}}}} \approx m_{0} c^{2}+\frac{1}{2} m_{0} v^{2}+\frac{3}{8} m_{0} \frac{v^{4}}{c^{2}}+\cdots \quad \text { with } v<c
$$

With Equation (17) and Equation (18), we modify Equation (5) to get Equation (21).

$$
m_{u}=\frac{m_{e} \sqrt{\beta}}{\alpha^{39}}=1.728098528(26) \times 10^{53} \mathrm{~kg}
$$

This equation is one of the most precise of all since it relies on very well-known constants defined in the CODATA 2014 [8]. It also highlights the fact that there are very strong links between the universe's dimensions and its constituents as such as the electron.

\subsection{Calculation of $m_{u}$ as a Function of the Classical Electron Charge $q_{e}$}

The electron charge $q_{e}$ may be described as a function of the electron mass $m_{e}$, the electron radius $r_{e}$ and the relative permeability of vacuum $\varepsilon_{0}$ [5].

$$
q_{e}=-\sqrt{\frac{4 \pi r_{e} m_{e}}{\mu_{0}}} \approx-1.60 \times 10^{-19} \mathrm{C}
$$

If we isolate the electron mass $m_{e}$ from Equation (22) and if we put its value in Equation (21), we get an equation that gives the apparent mass of the universe as a function of the electron charge $q_{e}$. Its precision is equivalent to Equation (21).

$$
m_{u}=\frac{\mu_{0} q_{e}^{2} \sqrt{\beta}}{4 \pi r_{e} \alpha^{39}}=1.728098528(26) \times 10^{53} \mathrm{~kg}
$$

\subsection{Calculation of $m_{u}$ as a Function of the Rydberg Constant $\boldsymbol{R}_{\infty}$}

Let us use the definition of the Rydberg constant $R_{\infty}$ as a function of the fine-structure constant $\alpha$ and the classical electron radius $r_{e}$ to find the apparent mass of the universe.

$$
R_{\infty}=\frac{\alpha^{3}}{4 \pi r_{e}} \approx 1.10 \times 10^{7} \mathrm{~m}^{-1}
$$


If we replace the classical electron radius $r_{e}$ from Equation (23) with Equation (24), we get Equation (25) that gives the apparent mass of the universe $m_{u}$ as a function of the Rydberg $R_{\infty}$ constant and the electron charge $q_{e}$.

$$
m_{u}=\frac{\mu_{0} q_{e}^{2} R_{\infty} \sqrt{\beta}}{\alpha^{42}}=1.728098528(27) \times 10^{53} \mathrm{~kg}
$$

\section{Apparent Radius of Curvature of the Universe $R_{u}$}

Let us enumerate different ways to calculate the apparent radius of curvature of the universe $R_{u}$.

We use the term "apparent" because the radius value is seen from our point of view in the universe. For an observer located somewhere else, the observed value may be different. Please refer to our section talking about the "quantum of speed" (further in this article) to deepen our notion of "appearance" for the different parameters of the universe. Also, we prefer to use the term "apparent radius of curvature of the universe" instead of talking about the "radius of curvature" of the universe as mentioned by Einstein [3], the "radius of the universe" [13] or the "radius of the space" [1] [13] like Lemaitre because the universe may looks spherical from our point of view (and we pretend that it is probably the case). However, what looks like a spherical shape is perhaps only local in the universe. Who knows, maybe the real shape of the universe is a peanut or a toroidal shape? The "apparent radius of curvature" is what we think it could be if we extrapolate the local characteristics and behaviors of the universe to a large scale. Of course, we assume here that the universe is a spherical, homogenous and isotropic. We highlight that the real radius of the universe may well be totally different if we consider other aspects of the universe which may become obvious at large scale.

Let us note that even if we get the same resulting distance as the Hubble radius [14] (which is seen as if the Earth were in the middle of this sphere), we measure here the distance between the center of mass of the universe and the outer limit of the luminous universe. In our model, the Earth is no more the "center of the universe". The universe is expanding. So, this limit is pushed further every day. The distance that light may travel within a year is only about $7 \times 10^{-9} \%$ of the total radius $R_{u}$.

\subsection{Calculation of $R_{u}$ as a Function of the Hubble Constant $H_{0}$}

The classical way to calculate the apparent radius of curvature of the universe $R_{u}$ consist in saying that the speed of light in vacuum $c$ was constant and traveled a time-lapse equal to an age of the universe $T_{u}$ which is a function of $H_{0}$ (see Equation (2)).

$$
R_{u}=c T_{u}=\frac{c}{H_{0}} \approx 1.28 \times 10^{26} \mathrm{~m}
$$

According to different sources, $H_{0}$ is between $67.8(9) \mathrm{km} \cdot \mathrm{s}^{-1} \cdot \mathrm{MParsec}^{-1}$ [15] and $77.6_{-4.3}^{+4.8} \mathrm{~km} \cdot \mathrm{s}^{-1} \cdot \mathrm{MParsec}^{-1}$ [16]. Most measurements of the Hubble constant $H_{0}$ rely on inaccurate methods such as the observation of stars. Uncertainties 
from different measurement results do not always overlap. For better accuracy, we will use $H_{0}$ from our Equation (18). It is compatible with Salvatelli, with $H_{0} \approx 72.1_{-2.3}^{+3.2} \mathrm{~km} \cdot \mathrm{s}^{-1} \cdot \mathrm{MParsec}^{-1}$ [9].

\subsection{Calculation of $R_{u}$ as a Function of the Classical Electron Radius $r_{e}$}

Let us use Equation (18) in Equation (26) to evaluate precisely (for metrology purposes) the apparent radius of curvature of the universe $R_{u}$.

$$
R_{u}=\frac{r_{e}}{\alpha^{19} \sqrt{\beta}} \approx 1.2831078845(57) \times 10^{26} \mathrm{~m}
$$

\subsection{Calculation of $\boldsymbol{R}_{u}$ as a Function of the Electron Charge $q_{e}$}

Let us define the apparent radius of curvature $R_{u}$ as a function of the Rydberg constant $R_{\infty}$. Let us isolate $r_{e}$ from Equation (22) and use it in Equation (27). We get Equation (28) which gives the apparent radius of curvature of the universe $R_{u}$ as a function of the electron charge $q_{e}$ and of the electron mass $m_{e}$.

$$
R_{u}=\frac{q_{e}^{2} \mu_{0}}{4 \pi m_{e} \alpha^{19} \sqrt{\beta}} \approx 1.283107889(13) \times 10^{26} \mathrm{~m}
$$

\subsection{Calculation of $\boldsymbol{R}_{u}$ as a Function of the Rydberg Constant $\boldsymbol{R}_{\infty}$}

Like Equation (28) which uses well-known parameters from CODATA and $\beta$ constant which can be calculated very accurately, we want to define the apparent radius of curvature $R_{u}$ as a function of the Rydberg constant $R_{\infty}$. Let us rewrite Equation (27) with Equation (24) to get the most precise way, up to now, to calculate the apparent radius of the universe $R_{u}$.

$$
R_{u}=\frac{1}{4 \pi R_{\infty} \alpha^{16} \sqrt{\beta}} \approx 1.2831078902(48) \times 10^{26} \mathrm{~m}
$$

\section{Age of the Universe $\Delta t_{u}$}

NASA currently estimates the age of the universe by using the inverse of the Hubble constant [17], that is to say, $1 / H_{0}$. The metric based on the work made by the physicists Friedmann [18] [19], Lemaitre [1] [13], Robertson [20] and Walker [21] predicts that for a flat universe dominated by the presence of matter, the true age of the universe should be around $2 /\left(3 \cdot H_{0}\right)$ [22]. Let us note that sometimes the name of Friedmann or Lemaitre is omitted when this metric is cited. This is the case in Carroll's book which refers to this metric under the name "FRW".

Einstein was considering the universe as being static [3], with constant space-time dimensions. When he first noticed that his theory of general relativity leads to a universe in expansion or in contraction, he added a cosmological constant to his equations to force his model of the universe to be static [3] Let's mention that in his theory of relativity, Einstein was taking for granted that the speed of light in the vacuum was constant [23] [24]. It was entirely consistent 
with his view of the universe. A static universe leads to a constant speed of light, except, of course, on the outskirts of large masses as is shown by Einstein with special relativity [24] and Schwarzschild with general relativity [25].

In 1929, Hubble found that the universe was expanding [4]. When Einstein became aware of Hubble's observations, he was forced to admit, according to George Gamow, that adding a cosmological constant to his model of the universe to make it static was the biggest blunder he has made in his life [26]. Let us note that Einstein may have never used these precise words and they may have been falsely reported from Gamow. Nevertheless, Einstein discarded his cosmological constant as an unnecessary fudge factor. It looks like he did not see, at that moment, that the acceleration of light over time was a direct result of an expanding universe. With recent work, we showed that it is possible that the speed of light has never been constant over time [5].

With special relativity, Einstein showed that a gravitational field generated by a mass slows down light [24]. Erroneous by a factor of 2 compared to what happens in reality, his equation, is then corrected by Schwarzschild using general relativity [25]. The universe is expanding [4], and becomes less dense. Therefore, the index of refraction diminishes and light slowly accelerates over time [5].

Initially, we will show how the approximate age of the universe is calculated. In a second step, we will use some results from a work we have done recently [5] to estimate the age of the universe by performing the integral of the inverse of the expansion speed of the material universe according to the curvature radius of the universe. Finally, we will approximate the age of the universe. We will show that $1 / H_{0}$ actually represents a good approximation of the apparent age $T_{u}$ of the universe and that $2 /\left(3 H_{0}\right)$ represents the real part of the age of the universe. We can then compare the results and comment.

\subsection{Current Methods for the Calculation of the Age of the Universe}

In 1929, Edwin Powell Hubble found that galaxies distance themselves from one another at a speed proportional to the distance between them [4]. He deduced a law involving a constant called $H_{0}$. It represents the average recession speed $v$ of galaxies per unit of distance $\Delta r$. Let us note that galaxies have their own freedom of movements. Some may move closer to each other and some will move away from each other. But overall, they will move away from each other because of the inflating movement of the universe.

$$
\frac{v}{\Delta r}=\frac{1}{\Delta t}=H_{0}=72.1 \mathrm{~km} \cdot \mathrm{MParsec}^{-1} \cdot \mathrm{s}^{-1}
$$

As seen in Equation (18), $H_{0} \approx 72.1 \mathrm{~km} \cdot \mathrm{MParsec}^{-1} \cdot \mathrm{s}^{-1}$. Let us isolate $\Delta t$.

$$
\Delta t=\frac{1}{H_{0}} \approx 13.65 \times 10^{9} \text { years }
$$

NASA is currently evaluating the age of the universe with Equation (31) [17]. This way of calculating the age assumes that the expansion rate of the universe is 
constant.

According to the model of Friedmann-Robertson-Walker [22], the true age of the universe would be around $2 / 3$ of Equation (31).

$$
\Delta t=\frac{2}{3 \cdot H_{0}} \approx 9.04 \times 10^{9} \text { years }
$$

\subsection{Calculation of the Age of the Universe $\Delta t_{u}$}

The speed of the light in vacuum and the expansion speed of the material universe may have not been constant over time [5]. Our goal here is to calculate the age of the universe (of complex type) by simulating a return to the past by doing the integral of the inverse of the expansion speed $v_{m}(r)$ of the material universe.

We will use some results of the work cited in [5] to solve the integral of the inverse of the speed $v_{m}(r)$ of expansion of the material universe as a function of the radius of curvature of the universe. In this way, we will calculate the age of the universe $\Delta t_{u}$.

As mentioned before, the general theory of relativity predicts that the presence of a massive body changes near space-time and increases the index of refraction $n(r)$ (which changes as a function of the distance $r$ from the center of mass of the massive body) of the vacuum around a mass [25]. By moving away from that mass, the gravitational influence is being reduced and the speed of light tends toward $c$.

We apply the same principle to the universe which is certainly the biggest existing mass. Since the universe is expanding [4], we move away from a certain center of mass and the density of the universe becomes lower over time. Like before, this causes the refractive index to diminish over time and lets the speed of light slowly increase over time tending toward an asymptotic speed that we named $k$ [5]. Of course, the current speed of light in vacuum is $c$. To keep the total energy of the universe constant meanwhile the speed of light increases, the mass of the universe must slowly decrease over time. The asymptotic speed of light $k$ (when $r \rightarrow \infty$ ) is given by Equation (33).

$$
k=c \sqrt{2+\sqrt{5}} \approx 2 c \approx 6.17 \times 10^{8} \mathrm{~m} \cdot \mathrm{s}^{-1}
$$

As soon as we try to calculate the speed of light for the time in the past or in the future, we must take into account that the speed of light $v_{L}(r)$ changes as a function of the apparent radius of curvature $r$ of the universe [5]. The value of $\Theta$ is the gravitational potential for the universe and $n(r)$ is the refractive index of the universe as a function of $r$.

$$
v_{L}(r)=\frac{k}{n(r)} \quad \text { where } n(r)=\sqrt{\frac{1-2 \Theta / k^{2}}{1+2 \Theta / k^{2}}} \text { and } \Theta=\frac{-G m_{u}}{r} \leq 0
$$

In this equation, there is a radius of curvature $r=r_{h}$ for which the speed of light $v_{L}\left(r_{h}\right)=0$. This position $r_{h}$ is called the horizon of the universe. This is the position for which the denominator of the square root of Equation (34) becomes 
zero. In a similar way, in a black hole, the radius of curvature of the horizon $r_{h}$ is obtained by the Schwarzschild's radius where we replace $c$ by $k$.

$$
r_{h}=\frac{2 G m_{u}}{k^{2}} \approx 6.06 \times 10^{25} \mathrm{~m}
$$

It is the same principle as that of a black hole. In fact, the universe is the biggest existing black hole since it has the biggest mass.

Let us note that for a conventional black hole, its entire mass is located in its center of mass. However, for the universe, a big part of the black hole mass lays outside the boundaries of the horizon. The center of mass of the universe coincides with the center of mass of the black hole.

The expansion speed of the universe is currently the speed of light $c$ [5]. Based on the principles of relativity, the matter cannot move at the speed of light without having infinite energy. Consequently, the previous assertion about the expansion of the universe can be true only for light (which we call the luminous universe). The material universe (containing the galaxies, intergalactic dust clouds, etc.) is expanding at a slower speed equal to $\beta c$. The factor $\beta$, must by necessity be less than 1 since we cannot surpass the speed of light which represents a speed boundary. According to our Equation (19), its value is about 0.76. The apparent radius of curvature $r_{u}$ of the material universe is, therefore, a portion $\beta$ of the apparent radius of curvature $R_{u}$ of the luminous universe [5].

$$
r_{u}=\beta R_{u} \approx 9.80 \times 10^{25} \mathrm{~m}
$$

The expansion speed $v_{m}(r)$ of the material universe is $\beta$ times the speed of light $v_{L}(r)$ because matter must travel slower than light [11].

$$
v_{m}(r)=\beta v_{L}(r)=\frac{\beta k}{n(r)} \text { where } n(r)=\sqrt{\frac{1-2 \Theta / k^{2}}{1+2 \Theta / k^{2}}} \text { and } \Theta=\frac{-G m_{u}}{r} \leq 0
$$

If we take the derivative of the expansion speed $v_{m}(r)$ of the material universe with respect to the distance $r$, we get the Hubble constant $H_{0}$ [5].

$$
\left.\frac{\mathrm{d} v_{m}(r)}{\mathrm{d} r}\right|_{r=r_{u}}=\frac{k \beta y}{r_{u}}\left(\frac{1}{(1+y) \sqrt{1-y^{2}}}\right)=H_{0} \quad \text { where } y=\frac{2 G m_{u}}{k^{2} r_{u}}
$$

It is important to realize that we do not use the derivative of the expansion speed of the luminous universe to get $H_{0}$ since astronomers cannot observe that limit. Through their telescopes, they only see objects like stars and galaxies. Consequently, when Hubble defined its constant $H_{0}$, it was based on the derivative of the expansion speed $v_{m}(r)$ of the material universe.

At the periphery of the luminous universe (at a distance $r=R_{u}$ from the center of mass of the universe), light accelerates at an $a_{L}\left(R_{u}\right)=c H_{0}$ rhythm.

$$
a_{L}\left(R_{u}\right)=\left.\frac{\mathrm{d} v_{L}(r)}{\mathrm{d} t}\right|_{r=R_{u}}=\left.\left(\frac{\mathrm{d} r}{\mathrm{~d} t} \cdot \frac{\mathrm{d} v_{L}}{\mathrm{~d} r}\right)\right|_{r=R_{u}}=\left.c\left(\frac{\mathrm{d} v_{L}}{\mathrm{~d} r}\right)\right|_{r=R_{u}}=c H_{0}
$$

However, locally, at a distance $r=r_{u}$ from the center of mass of the universe, light slowly accelerates at an $a_{L}\left(r_{u}\right)=c H_{0} / \beta$ rhythm. 


$$
a_{L}\left(r_{u}\right)=\left.\frac{\mathrm{d} v_{L}(r)}{\mathrm{d} t}\right|_{r=r_{u}}=\left.\left(\frac{\mathrm{d} r}{\mathrm{~d} t} \cdot \frac{\mathrm{d} v_{L}}{\mathrm{~d} r}\right)\right|_{r=r_{u}}=\left.c\left(\frac{\mathrm{d} v_{L}}{\mathrm{~d} r}\right)\right|_{r=r_{u}}=c \frac{H_{0}}{\beta}
$$

Locally, at a distance $r=r_{u}$ from the center of mass of the universe, matter from the material universe slowly accelerates at an $a_{m}\left(r_{u}\right)=c H_{0}$ rhythm.

$$
a_{m}\left(r_{u}\right)=\left.\beta \frac{\mathrm{d} v_{L}(r)}{\mathrm{d} t}\right|_{r=r_{u}}=\left.\beta\left(\frac{\mathrm{d} r}{\mathrm{~d} t} \cdot \frac{\mathrm{d} v_{L}}{\mathrm{~d} r}\right)\right|_{r=r_{u}}=\left.\beta c\left(\frac{\mathrm{d} v_{L}}{\mathrm{~d} r}\right)\right|_{r=r_{u}}=\beta c \frac{H_{0}}{\beta}=c H_{0}(41)
$$

Figure 1 shows the module of the expansion speed $v_{m}(r)$ of the material universe calculated from Equation (37). It also puts in evidence the Hubble constant $H_{0}$ as the slope evaluated precisely at the position $r=r_{u}$ from the center of mass of the universe (at $r=0$ ). Between $r=0$ (at the Big Bang) and $r=r_{h}$ (horizon of the universe), the dotted part of the curve shows that the expansion speed of the material universe would normally be negative and of imaginary type. Then, from $r=r_{h}$ and infinite, the expansion speed of the material universe becomes of real type.

The negative and imaginary value of the speed $v_{m}(r)$ we get with $r<r_{h}$ is a mathematical way to show that in a physical world, matter inside the horizon evolves independently than outside the horizon. Here, we cannot see what is happening inside the horizon and vice versa. We could compare it to the cylindrical envelope of an optical fiber. Light can travel inside a fiber from one extremity to the other end, but we cannot see through the envelope (if the fiber is used in its curving limits). It is therefore impossible to know what is happening from outside the fiber and vice versa. Of course, the principles in cause in fiber are not the same. We only bring this example to do an image of the situation happening inside the horizon of a black hole.

Performing the integral of the inverse of $v_{m}(r)$ with respect to the radius of curvature $r$, it is possible to calculate precisely the age of the universe more precisely (in its entirety with the real part and its imaginary part) than by using a single tangential projection. In Figure 1, the slope of the tangential projection gives the Hubble constant $H_{0}$ which can be put in Equation (31) to give the apparent age of the universe.

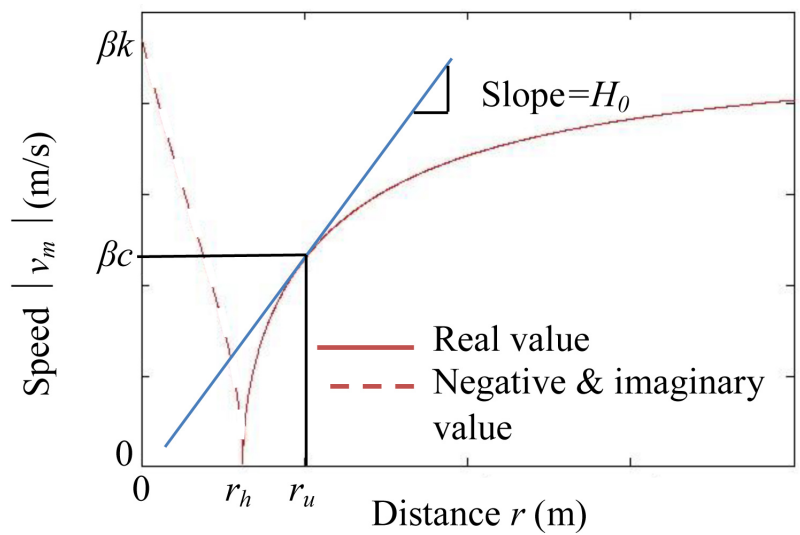

Figure 1. Expansion speed $\left|v_{m}\right|$ of the material universe as a function of distance $r$. 
Let's find the age of the universe $\Delta t_{u}(r)$ by performing the integral of Equation (42) between the center of mass of the universe (at $r=0$ ) and the apparent radius of curvature of the material universe $r_{u}$. The resulting value $\Delta t_{u}(r)$ is of a complex type.

$$
\Delta t_{u}(t)=\int_{0}^{r_{u}} \frac{1}{v_{m}(r)} \mathrm{d} r=\Delta t_{h u}(r)+\Delta t_{0 h}(r)
$$

In Equation (42), the value of $\Delta t_{h u}(r)$ represents the time elapsed between the horizon and the actual age of the universe (see Equation (43)). The resulting value is of real type.

$$
\Delta t_{h u}(r)=\int_{r_{h}}^{r_{u}} \frac{1}{v_{m}(r)} \mathrm{d} r
$$

In Equation (42), the value of $\Delta t_{0 h}(r)$ represents the time elapsed between the Big Bang and the horizon (see Equation (44)). The resulting value is of an imaginary type.

$$
\Delta t_{0 h}(r)=\int_{0}^{r_{h}} \frac{1}{v_{m}(r)} \mathrm{d} r
$$

Performing the integral calculation of Equation (42), we get Equation (45).

$$
\begin{aligned}
& \int \frac{1}{v_{m}(r)} \mathrm{d} r=\frac{\left(z(r)+2 G \cdot m_{u} \ln \left(2\left[k^{2} r+z(r)\right]\right)\right)}{\beta k^{3}} \\
& \text { where } z(r)=\sqrt{k^{4} r^{2}-4 G^{2} m_{u}^{2}}
\end{aligned}
$$

Consequently, the value of $\Delta t_{u}(r)$ becomes:

$$
\Delta t_{u}(r)=\Delta t_{h u}(r)+\Delta t_{0 h}(r) \approx(9.50+10.47 i) \times 10^{9} \text { years where } i=\sqrt{-1}
$$

This result is of a complex type. In Equation (46), the first part of the integral (shown in Equation (43)) is of a real type (between $r_{h}$ and $r_{u}$ ). However, the second part of this latter one (shown in Equation (44)) is of an imaginary type (between 0 and $r_{h}$ ).

If we look carefully to Equation (37), for a radius smaller than the horizon $r_{h}$, the speed of light $v_{L}\left(r_{h}\right)$ becomes of an imaginary type. For this reason, the time $T_{0 h}$ become of an imaginary type as well. This mathematical situation just means that time inside the horizon evolves in a completely independent way compared to time outside the horizon, which is why no one can see what happens inside the limits of the horizon of a black hole. The best example we could give is within a fiber optic cable. How light evolves inside the fiber cannot be seen from outside the fiber, and vice versa. Mathematics are a nice language which has to be interpreted to find sense in the real world.

When we wish to consider the time elapsed between position 0 of the Big Bang and the radius of curvature of the horizon $r_{h}$, we must calculate the module of the time elapsed $\left|\Delta t_{u}\right|$. We define this value as the apparent age $T_{u}$ of the universe because it does not necessarily represent the true age of the universe. This 
number represents only an apparent age in the likely event that the Big Bang existed. It could also be seen as the time that elapsed if the speed of light had been constant over time since the Big Bang.

$$
T_{u}=\left|\Delta t_{u}\right|=\left|\Delta_{h u}+\Delta t_{0 h}\right|=\sqrt{\Delta t_{h u}^{2}+\Delta t_{0 h}^{2}} \approx 14.14 \times 10^{9} \text { years }
$$

We see that the value is only $4.25 \%$ over the value of Equation (31).

\subsection{Approximation of the Age of the Universe}

As for the calculation of power in electrical motors (with the real power, the inductive power and the apparent power), the age of the universe may be seen as follows: the "real" part of the age of the universe, the "imaginary" part of the age of the universe and the "apparent" age of the universe. The module of the two components (real and imaginary) can be calculated using the Pythagorean Theorem by finding the square root of the sum of the squares of the real part and the imaginary part of the age of the universe.

The approximation of the age of the universe will be made in 3 parts: the approximation of the real part of the age of the universe, the approximation of the imaginary part of the age of the universe and the calculation of the module of the apparent age of the universe. In Figure 2, we show the parallelogram built from these values.

\subsection{Approximation of the Real Part $\Delta t_{h u}$ of the Age of the Universe}

Let's perform the approximation of the real part $\Delta t_{h u}$ of the age of the universe $\Delta t_{u}$

For a radius of curvature $r_{h}$ of the horizon, the square root in Equation (48) equals zero.

$$
z\left(r_{h}\right)=\sqrt{k^{4} r_{h}^{2}-4 G^{2} m_{u}^{2}}=0
$$

So, according to Equation (42) and Equation (45), we get Equation (49).

$$
\begin{aligned}
& \Delta t_{h u}=\frac{z\left(r_{u}\right)+2 \cdot G \cdot m_{u} \cdot \ln \left(2 \cdot\left[k^{2} \cdot r_{u}+z\left(r_{u}\right)\right]\right)}{\beta \cdot k^{3}}-\frac{2 \cdot G \cdot m_{u} \cdot \ln \left(2 \cdot k^{2} \cdot r_{h}\right)}{\beta \cdot k^{3}} \\
& \text { where } z\left(r_{u}\right)=\sqrt{k^{4} \cdot r_{u}^{2}-4 \cdot G^{2} \cdot m_{u}^{2}}
\end{aligned}
$$

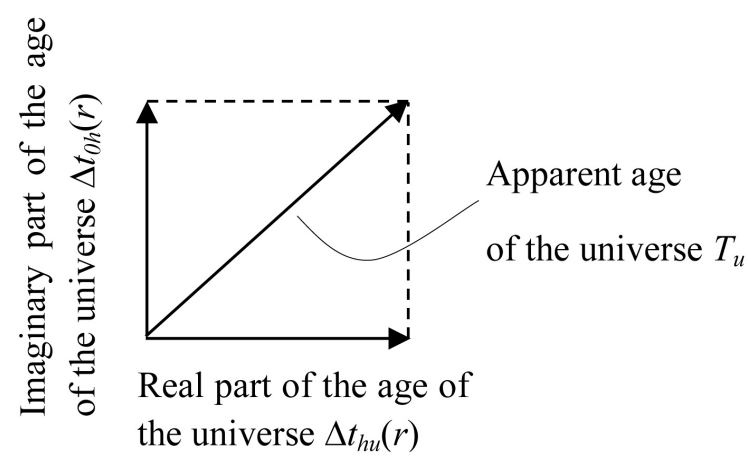

Figure 2. Complex age $\Delta t_{u}$ of the universe. 
Using Equation (35) with a few simplifications, we get Equation (50).

$$
\Delta t_{h u}=\frac{1}{\beta \cdot k}\left(\sqrt{r_{u}^{2}-r_{h}^{2}}+r_{h} \ln \left(\frac{\sqrt{r_{u}^{2}-r_{h}^{2}}+r_{u}}{r_{h}}\right)\right)
$$

Without changing anything, this same equation could be rewritten as follows.

$$
\begin{aligned}
& \Delta t_{h u}=\frac{r_{u}}{2 \cdot \beta \cdot c} \cdot\left(1+\frac{\beta}{2}\right) \cdot y \approx \frac{r_{u}}{2 \cdot \beta \cdot c} \cdot\left(1+\frac{\beta}{2}\right) \\
& \text { where } y=\left[\frac{2 \cdot c}{k \cdot r_{u} \cdot\left(1+\frac{\beta}{2}\right)} \cdot\left(\sqrt{r_{u}^{2}-r_{h}^{2}}+r_{h} \ln \left(\frac{\sqrt{r_{u}^{2}-r_{h}^{2}}+r_{u}}{r_{h}}\right)\right)\right] \approx 1
\end{aligned}
$$

As shown, the content of the bracket is approximately equal to 1 . By doing this approximation and using Equation (36), Equation (51) becomes Equation (52).

$$
\Delta t_{h u} \approx \frac{1}{4 H_{0}}(2+\beta)
$$

Further, in this document, we will use Equation (52) to perform the calculation of the apparent age of the universe. For now, let us show that this equation can be approximated to get an equation commonly used by some astronomers to calculate the actual age of the universe.

According to Equation (19), the value of $\beta \approx 0.76$. Let's use this approximation to rewrite Equation (52). After a few simplifications, we get:

$$
\Delta t_{h u} \approx \frac{1}{4 H_{0}}\left(2+\frac{76}{100}\right) \approx \frac{1}{H_{0}}\left(\frac{69}{100}\right) \approx \frac{2}{3 H_{0}} \approx 9.04 \times 10^{9} \text { years }
$$

This last ratio can be deduced from the model of Friedmann-Robertson-Walker [22]. Therefore, Equations (52) and (53) represent good approximations of the real part of the age of the universe.

\subsection{Approximation of the Imaginary Part $\Delta t_{0 h}$ of the Age of the Universe}

Now, let's find the approximate value of the imaginary part $T_{0 h}$ of the age of the universe $T_{u}$. From Equation (42), Equation (45) and Equation (48) we get:

$$
\Delta t_{0 h}=\left[\frac{2 \cdot G \cdot m_{u} \cdot \ln \left(2 \cdot k^{2} \cdot r_{u}\right)}{\beta \cdot k^{3}}\right]-\left[\frac{\sqrt{-4 \cdot G^{2} \cdot m_{u}^{2}}+2 \cdot G \cdot m_{u} \cdot \ln \left(2 \cdot \sqrt{-4 \cdot G^{2} \cdot m_{u}^{2}}\right)}{\beta \cdot k^{3}}\right]
$$

Using Equation (35) and performing a few simplifications, we get Equation (55).

$$
\Delta t_{0 h}=\frac{r_{h}}{\beta \cdot k} \cdot[\ln (-i)-i] \text { where } i=\sqrt{-1}
$$

Using the relationship in Equation (56), we rewrite Equation (55), which is the result of a purely imaginary type, to get Equation (57). 


$$
\begin{gathered}
\ln (-i)=-\frac{\pi}{2} \cdot i \\
\Delta t_{0 h}=-\frac{r_{h}}{\beta \cdot k} \cdot\left[1+\frac{\pi}{2}\right] \cdot i
\end{gathered}
$$

We can rewrite Equation (57) in the following way without changing anything:

$$
\Delta t_{0 h}=-\frac{r_{u}}{2 \cdot \beta \cdot c} \cdot\left(1-\frac{\beta}{2}\right) \cdot\left(1+\frac{\pi}{2}\right) \cdot x \cdot i \text { where } x=\left[\frac{2 \cdot c}{k} \cdot \frac{r_{h}}{r_{u} \cdot\left(1-\frac{\beta}{2}\right)}\right] \approx 1
$$

As shown, the value of $x$ is approximately 1. Equation (58) then becomes:

$$
\Delta t_{0 h} \approx-\frac{1}{2 \cdot H_{0}} \cdot\left(1-\frac{\beta}{2}\right) \cdot\left(1+\frac{\pi}{2}\right) \cdot i
$$

\subsection{Approximation of the Apparent Age $T_{u}$ of the Universe}

Let's calculate the apparent age of the universe by using Equation (47) with Equation (52) and Equation (59). After a few simplifications, we get Equation (60).

$$
\begin{aligned}
& \left|\Delta t_{u}\right|=\left|\Delta t_{h u}+\Delta t_{0 h}\right|=\sqrt{\Delta t_{h u}^{2}+\Delta t_{0 h}^{2}} \approx \frac{1}{H_{0}} \chi \approx \frac{1}{H_{0}} \\
& \text { where } \chi=\left(\frac{1}{2} \cdot \sqrt{\left(1+\frac{\beta}{2}\right)^{2}+\left(\left(1-\frac{\beta}{2}\right) \cdot\left(1+\frac{\pi}{2}\right)\right)^{2}}\right) \approx 1
\end{aligned}
$$

The value of $\chi$ is approximately equal to 1 . Consequently, we have shown that the integral of Equation (42) can be approximated by Equation (31). According to us, based on the approximation calculation made at Equation (60), Equation (31) represents only an apparent age $T_{u}$ of the universe. In fact, it comes from the calculation of the module of a complex sum of the real part and the imaginary part of the age of the universe. We define $T_{u}$ as the apparent age of the universe.

$$
T_{u}=\left|\Delta t_{u}\right| \approx \frac{1}{H_{0}} \approx 13.65 \times 10^{9} \text { years }
$$

\section{Quantum of Speed $\varepsilon_{v}$}

By definition, a quantum (the word "quanta" is plural) represents the smallest indivisible amount of any physical entity. For example, Planck length $L_{p}$ represents a quantum of distance and Planck time $t_{p}$ represents a quantum of time. The quantum of speed is the smallest variation of speed that can be obtained. It is so small that it is immeasurable. Nevertheless, it is possible to calculate it thanks to certain physical considerations.

We want to show, among other things, that the different parameters (apparent mass, radius, and age) of the universe are what they are from our point of view. However, they may look different from the point of view of an observer traveling 
at the speed of light. Thus, everyone will understand why we consider these parameters as being "apparent".

In relativity, the speed of light in vacuum $c$ is considered the speed limit. It is used in the Lorentz factor. We will show that even light does not exactly travel at the speed limit $\mathcal{c}$, but a bit less. In fact, the real speed of light is $c$ - $\mathcal{E}_{V}$ where $\varepsilon_{V}$ is what we call the quantum of speed. This is the smallest variation of speed that can be measured.

Let us suppose an observer at rest looking at a mass $m_{0}$. According to special relativity [11] [27], if we accelerate the mass at a speed $v$, for the observer, the mass becomes $m^{\prime}$.

$$
m^{\prime}=\frac{m_{0}}{\sqrt{1-\frac{v^{2}}{c^{2}}}}
$$

We would be tempted to say that when $v \rightarrow c$, the mass $m^{\prime}$ tends towards infinity. However, this is not logical since it is impossible to reach a mass bigger than the mass of the universe $m_{u}$. We cannot give to a mass more energy than what is available in the whole universe. This statement imposes a new limit to the speed $v$.

We make the statement that Planck mass $m_{p}$ represents the highest level of energy for a particle. It is easy to verify this assertion by equating the energy of an arbitrary mass $m$ with the energy of the smallest wavelength $\lambda$ possible (which is $2 \pi L_{p}$, where $L_{p}$ is the Planck length). Planck length $L_{p}$ is considered, in a quantum world, the smallest unit of length. This is due to the Heisenberg's uncertainty principle which says that we cannot measure precisely the speed of an object and its precise location at the same time [28]. Here, the mass-energy from a particle (given by Einstein's equation [11] [27] $E=m c^{2}$ ) is associated to the wave energy (given by Planck's formula $E=h c / \lambda[29]$ ).

$$
m c^{2}=\frac{h c}{\lambda} \quad \text { where } \lambda=2 \pi L_{p}
$$

The standard definition of a Planck length is Equation (64), which implies the universal gravitational constant $G$, the Planck constant $h$ and the speed of light in vacuum $c$.

$$
L_{p}=\sqrt{\frac{h G}{2 \pi c^{3}}} \approx 1.62 \times 10^{-35} \mathrm{~m}
$$

If we replace $L_{p}$ in Equation (63) and if we isolate the mass $m$, we get Equation (65) which is exactly the standard definition of Planck mass $m_{p}$.

$$
m=\sqrt{\frac{h c}{2 \pi G}}=m_{p} \approx 2.18 \times 10^{-8} \mathrm{~kg}
$$

From this statement, we can calculate the maximum speed $V_{m}$ at which we might move a particle having an initial mass $m_{p h}$ at rest. We give to this mass the same value as the mass associated to a photon of wavelength $\lambda=2 \pi R_{u}$ (the apparent circumference of the universe). Since this wavelength is the longest that 
we can reach in the universe, it corresponds to the lowest amount of energy we can get.

$$
\frac{m_{p h}}{\sqrt{1-\frac{v_{m}^{2}}{c^{2}}}}=m_{p} \rightarrow v_{m}=c \sqrt{1-\frac{m_{p h}^{2}}{m_{p}^{2}}}
$$

Since $m_{p h} \ll m_{p}$, we can make the following approximation.

$$
v_{m} \approx c\left(1-\frac{m_{p h}^{2}}{2 m_{p}^{2}}\right)
$$

In Equation (67), $m_{p h}$ is defined by Equation (11).

Dirac made the hypothesis that all large, dimensionless numbers that could be constructed from the important natural units of cosmology and atomic theory were connected. Let us call $N$ the ratio between the apparent mass of the universe $m_{u}$ and the mass $m_{p h}$ of associated with the photons of the lowest energy (from Equation (11)).

$$
N=\frac{m_{u}}{m_{p h}} \approx 6.30 \times 10^{121}
$$

Let us show that Equation (67) can be defined as a function of $N$. Let us rewrite Equation (68) with Equation (5) and Equation (11). Then, using the Planck mass $m_{p}$ (given by Equation (10)), we show that $N$ can be redefined as a function of $m_{p}$ and $m_{p h}$ (instead of $m_{u}$ and $m_{p h}$ ).

$$
N=\frac{c^{3}}{G H_{0}} \cdot \frac{2 \pi R_{u} c}{h}=\frac{h c}{2 \pi G} \cdot \frac{4 \pi^{2} R_{u}^{2} c^{4}}{h^{2} c^{2}}=\frac{m_{p}^{2}}{m_{p h}^{2}} \approx 6.30 \times 10^{121}
$$

Let's define the speed $v_{m}$ in Equation (67) as a function of $N$ with Equation (69).

$$
v_{m} \approx c-\frac{c}{2 N}
$$

If we replace $V_{m}$ by $c-\varepsilon_{b}$ the value of $\varepsilon_{v}$ could be defined as the "quantum of speed".

$$
\varepsilon_{v} \approx \frac{c}{2 N} \approx 2.38 \times 10^{-114} \mathrm{~m} \cdot \mathrm{s}^{-1}
$$

This speed variation $\mathcal{E}_{V}$ is the smallest speed unit possible. For an academic purpose, let us rewrite Equation (62) by replacing the speed $v$ per $c-n \varepsilon_{v}$ where $n$ is an integer $>0$.

$$
m^{\prime}=\frac{m_{0}}{\sqrt{1-\frac{v^{2}}{c^{2}}}} \text { where } v=c-n \varepsilon_{v} \text { and } n=1,2,3, \cdots, 2 N
$$

We see that with Equation (72), we cannot reach an infinite mass anymore and that the real speed of light is at least a quantum of speed slower than the speed limit $c$. Everyone will admit that $\varepsilon_{v}$ is a so small value compared to any 
speed value that it may be neglected most of the time. Nevertheless, it allows putting in evidence some speed boundaries.

The universe is expanding with relativistic speeds. For an observer traveling at the speed of light $\left(c-\mathcal{E}_{v}\right)$, the total mass of the universe would be much smaller. In fact, it would be seen as being only the Planck mass $m_{p} \approx 2.18 \times 10^{-8} \mathrm{~kg}$. If we travel at the same speed as a photon, we have to null out the Lorentz factor that is included at the denominator in the apparent mass of the universe $m_{u}$ to see what is happening from the observer point of view. We multiply $m_{u}$ by the Lorentz factor using Equations (70) and (71).

$$
m_{u} \sqrt{1-\frac{\left(c-\varepsilon_{v}\right)^{2}}{c^{2}}} \approx m_{u} \sqrt{\frac{1}{N}} \approx m_{p} \approx 2.18 \times 10^{-8} \mathrm{~kg}
$$

Planck mass $m_{p}$ is usually defined by Equation (65).

Similarly, for an observer traveling at the speed of light $\left(c-\varepsilon_{v}\right)$, the apparent radius of curvature $R_{u}$ would look like being only the Planck length $L_{p} \approx 1.6 \times$ $10^{-35} \mathrm{~m}$

$$
R_{u} \sqrt{1-\frac{\left(c-\varepsilon_{v}\right)^{2}}{c^{2}}} \approx R_{u} \sqrt{\frac{1}{N}} \approx L_{p} \approx 1.62 \times 10^{-35} \mathrm{~m}
$$

Planck length $L_{p}$ is usually defined by Equation (64).

Again, for an observer traveling at the speed of light $\left(c-\varepsilon_{v}\right)$, the apparent age of the universe $T_{u}$ would look like being only the Planck time $t_{p}$.

$$
T_{u} \sqrt{1-\frac{\left(c-\varepsilon_{v}\right)^{2}}{c^{2}}} \approx \frac{1}{H_{0}} \sqrt{1-\frac{\left(c-\varepsilon_{v}\right)^{2}}{c^{2}}} \approx \frac{1}{H_{0}} \sqrt{\frac{1}{N}} \approx t_{p} \approx 5.39 \times 10^{-44} \mathrm{~s}
$$

Planck time $t_{p}$ is usually defined by Equation (76).

$$
t_{p}=\sqrt{\frac{h G}{2 \pi c^{5}}}=\frac{L_{p}}{c} \approx 5.39 \times 10^{-44} \mathrm{~s}
$$

On another side, for an observer at rest, if there were no expansion, no movement, and no rotation in the universe, the total mass of the universe would be only the Planck mass $m_{p}$. Most of the universe energy (therefore its mass) is coming from different sort of relativistic movement. The demonstration becomes the same as in Equation (73). Obviously, if there were no expansion, the apparent radius of the universe would be the Planck length $L_{p}$. If there were no expansion in the universe, the apparent age of the universe would be the Planck time $t_{p}$

\section{Different Links between the Universe Dimensions}

Dirac made the hypothesis that all large, dimensionless numbers that could be constructed from the important natural units of cosmology and atomic theory were connected [6] [7]. Let us see some about $m_{u}$ (apparent mass of the universe), $m_{p}$ (Planck mass), $R_{u}$ (apparent radius of the universe), $L_{p}$ (Planck Length), $T_{u}$ (apparent age of the universe), $t_{p}$ (Planck time) and $H_{0}$ (Hubble con- 
stant).

$$
\begin{gathered}
N=\frac{m_{u}}{m_{p h}}=\frac{m_{u}^{2}}{m_{p}^{2}}=\frac{m_{p}^{2}}{m_{p h}^{2}}=\frac{m_{u} R_{u}}{m_{p} L_{p}}=\frac{m_{p} R_{u}}{m_{p h} L_{p}}=\frac{m_{u} T_{u}}{m_{p} t_{p}}=6.3 \times 10^{121} \\
N=\frac{m_{p} T_{u}}{m_{p h} t_{p}}=\frac{m_{u}}{m_{p} t_{p} H_{0}}=\frac{m_{p}}{m_{p h} t_{p} H_{0}}=\frac{R_{u}^{2}}{L_{p}^{2}}=\frac{T_{u}^{2}}{t_{p}^{2}}=\frac{1}{t_{p}^{2} H_{0}^{2}} \approx 6.3 \times 10^{121}
\end{gathered}
$$

Let us remind that $N$ represents the maximum number of photons of the lowest energy that may exist in the universe (if we were converting the entire mass of the universe in photons having a wavelength equal to the apparent circumference of the universe $\left.2 \pi R_{u}\right)$.

In Equation (77) and Equation (78), the values of the different parameters of the universe may be obtained from imprecise sources. A precise link between the large number $N[5]$ and the fine-structure constant $\alpha$ can be made.

$$
N=\frac{1}{\alpha^{57}} \approx 6.303419702(84) \times 10^{121}
$$

By showing Equations (77)-(79), our goal was to highlight the fact that there are tight and precise links between the infinitely large and infinitely small. Once we are aware of these interesting links, we can find more similar precise links with the large number $N$. More than a hundred other equations may be made about this large number $N$ and various parameters of the universe (temperature, charge, etc.) [30]. From a reverse way, we can get precise values for different parameters of the universe by equating Equation (79) with other equations giving $N$.

\section{Conclusions}

In this article, we have shown different ways to calculate the apparent mass of the universe, the apparent radius of curvature of the universe and the age of the universe. We also made the calculation of the quantum of speed. With these parameters, we used Dirac's large numbers hypothesis to show that there are links between all these parameters.

We have defined a new concept that we think must be introduced in physics: the "quantum of speed" $\mathcal{E}_{r}$. The "quantum of speed" notion made us conscious that there is a little difference between the real speed of light and the speed limit. For most application, it does not matter to say that both speeds are equal. Although, in some special case, it is necessary to put in evidence the difference. Using common sense, we show that it is evident that we cannot give more energy to any mass than there is energy in the whole universe (which is $m_{u}$ ). Also, since Planck mass $m_{p}$ is associated to the highest level of energy for an accelerated particle, we cannot give more energy to any particle than what is contained in the Planck mass $m_{p}$. Following these two findings, the "quantum of speed" $\varepsilon_{v}$ is naturally introduced.

It was a necessity to introduce the "quantum of speed" to be able to calculate what would be the apparent mass of the universe, the apparent radius of curva- 
ture and the age of the universe from the point of view of an observer traveling at the speed of light. Surprisingly, the results are totally different than what common sense would lead to. Only relativity allows us to interpret correctly the results according to the point of view of the observer.

From a metrology point of view, we reach our goal by obtaining precise values for different dimensions of the universe. Using these values will make it easier to see the different links we can make between large numbers of Dirac and the infinitely small. With imprecise values, we can pass beside nice occasions to make rise new theories.

\section{Conflicts of Interest}

The author declares no conflicts of interest regarding the publication of this paper.

\section{References}

[1] Kragh, H. (2012) Astrophysics and Space Science Library, 395, 23-38. https://doi.org/10.1007/978-3-642-32254-9_3

[2] Kragh, H. (2013) Astronomy \& Geophysics, 54, 228-230. https://doi.org/10.1093/astrogeo/att035

[3] Einstein, A. (1917) Kosmologische Betrachtungen zur allemeinen Relativitätstheorie. Springer Link, 130-139. https://doi.org/10.1007/978-3-663-19510-8_9

[4] Hubble, E. (1929) Proceedings of the National Academy of Sciences of the United States of America, 15, 168-1973. https://doi.org/10.1073/pnas.15.3.168

[5] Mercier, C. (2019) Journal of Modern Physics, 10, 641-662. https://doi.org/10.4236/jmp.2019.106046

[6] Dirac, P.A.M. (1938) Proceedings of the Royal Society of London A: Mathematical, Physical and Engineering Sciences, 165, 199-208. https://doi.org/10.1098/rspa.1938.0053

[7] Dirac, P.A.M. (1974) Proceedings of the Royal Society of London A: Mathematical, Physical and Engineering Sciences, 338, 439-446. https://doi.org/10.1098/rspa.1974.0095

[8] Mohr, P.J., Newell, D.B. and Taylor, B.N. (2016) Journal of Physical and Chemical Reference Data, 45, Article ID: 043102. https://doi.org/10.1063/1.4954402

[9] Salvatelli, V., Andrea, M., Laura, L.-H. and Olga, M. (2013) Physical Review D, 88, Article ID: 023531. https://doi.org/10.1103/PhysRevD.88.023531

[10] Carvalho, J.C. (1995) International Journal of Theoretical Physics, 34, 2507-2509. https://doi.org/10.1007/BF00670782

[11] Einstein, A. (1956) Physics Today, 9, 30. https://doi.org/10.1063/1.3059795

[12] Newton, I. (1686) Philosophiae Naturalis Principia Mathematica. Samuel Pepys and the Royal Society Praeses, London, 510. https://doi.org/10.5479/sil.52126.39088015628399

[13] Lemaître, G. (2013) General Relativity and Gravitation, 45, 1635-1646.

[14] Zichichi, A. (2000) Proceedings of the International School of Subnuclear Physics, 36, 708. https://doi.org/10.1142/9789812793935

[15] Ade, P.A.R., et al. (2016) Astronomy \& Astrophysics, 594, A13. 
https://doi.org/10.1051/0004-6361/201525830

[16] Bonamente, M., Joy, M.K., La Roque, S.J. and Carlstrom, J.E. (2005) The Astrophysical Journal, 647, 25-54. https://doi.org/10.1086/505291

[17] Jarosik, N. (2011) The Astrophysical Journal Supplement Series, $192,17$. https://doi.org/10.1088/0067-0049/192/2/14

[18] Friedmann, A. (1922) Zeitschrift für Physik, 10, 377-386. https://doi.org/10.1007/BF01332580

[19] Friedmann, A. (1924) Zeitschrift für Physik, 21, 326-332. https://doi.org/10.1007/BF01328280

[20] Robertson, H.P. (1933) Reviews of Modern Physics, 5, 62-90. https://doi.org/10.1103/RevModPhys.5.62

[21] Walker, A.G. (1937) Proceedings of the London Mathematical Society, 42, 90-127. https://doi.org/10.1112/plms/s2-42.1.90

[22] Carroll, S.M. (2004) Spacetime and Geometry: An Introduction to General Relativity. Addison Wesley, Boston, 513.

[23] Einstein, A. (1916) Annalen der Physik, 49, 769-822. https://doi.org/10.4324/9780203198711

[24] Einstein, A. (1911) Annalen der Physik, 35, 898-908. https://doi.org/10.1002/andp.19113401005

[25] Grøn, Ø. (2016) American Journal of Physics, 84, 537. https://doi.org/10.1119/1.4944031

[26] Weinberg, S. (2005) Physics Today, 58, 31-35. https://doi.org/10.1063/1.2155755

[27] Einstein, A. (1905) Annalen der Physik, 322, 891-921. https://doi.org/10.1002/andp.19053221004

[28] Heisenberg, W. (1927) Zeitschrift für Physik, 43, 172-198. https://doi.org/10.1007/BF01397280

[29] Planck, M. (1899) Natuerliche Masseinheiten. Der Königlich Preussischen Akademie Der Wissenschaften, 479.

[30] Mercier, C. (2016) More than a Hundred Ways to Get the Large Number N. Hypothesis and Thoughts on the Universe, 14.

http://www.pragtec.com/physique/index_en.html 